\title{
JOINING POLYNOMIAL AND EXPONENTIAL COMBINATORICS FOR SOME ENTIRE MAPS
}

\author{
Antonio Garijo, Xavier Jarque, and Mónica Moreno Rocha
}

\begin{abstract}
We consider families of entire transcendental maps given by $F_{\lambda, m}(z)=\lambda z^{m} \exp (z)$ where $m \geq 2$. All these maps have a superattracting fixed point at $z=0$ and a free critical point at $z=-m$. In parameter planes we focus on the capture zones, i.e., we consider $\lambda$ values for which the free critical point belongs to the basin of attraction of $z=0$. We explain the connection between the dynamics near zero and the dynamics near infinity at the boundary of the immediate basin of attraction of the origin, thus, joining together exponential and polynomial behaviors in the same dynamical plane.
\end{abstract}

\section{Introduction}

In this paper, we combine symbolic dynamics with polynomial-like theory to investigate the combinatorics of the Julia set of the families of transcendental entire functions

$$
F_{\lambda, m}(z)=\lambda z^{m} e^{z}
$$

with $m \geq 2$ and $\lambda \in \mathbb{C} \backslash\{0\}$. The Julia set of an entire map $f, J(f)$, is the set of points where the family of iterates $\left\{f^{n}\right\}$ fails to be a normal family. Its complement in $\mathbb{C}$ is an open set of the plane known as Fatou set, where the dynamics is tame.

For all functions in (1.1), 0 is a critical and asymptotic value and $F_{\lambda, m}(-m)=\lambda(-m)^{m} \exp (-m)$ is a critical value. Therefore, these maps belong to a general class of entire transcendental maps with only finitely many critical and asymptotic values also known as critically finite. The interest on critically finite maps resides in that they resemble rational maps, as their Fatou set contains neither wandering nor Baker domains (see $[\mathbf{2}],[\mathbf{1 4}],[\mathbf{1 5}]$ and $[\mathbf{1 7}])$. In contrast, the point at infinity plays a

2000 Mathematics Subject Classification. 37F20, 30D20.

Key words. Julia sets, polynomial-like maps, combinatorial dynamics. 
crucial role. For instance, Picard's Little Theorem says that an entire function in any neighborhood of infinity assumes infinitely many times each value in the complex plane with at most one exception.

The families $F_{\lambda, m}$ have been previously considered in the literature. In [4], Bergweiler considers functions related to $F_{\lambda, m}$ to provide examples of a Baker domain at a positive distance from any singular orbit. In [16], Fagella and the first author present a thorough analysis of the topology of capture zones in parameter plane and of Julia sets in dynamical plane.

As the exponential family $E_{\lambda}(z)=\lambda e^{z}$ is the transcendental version of the quadratic family $Q_{c}(z)=z^{2}+c$, the family $F_{\lambda, 2}$ is the transcendental version of the one-parameter slice of the cubic family $z \mapsto z^{3}-3 a^{2} z+b$, given by

$$
M_{a}(z)=z^{3}-\frac{3}{2} a z^{2} .
$$

We review some of its properties here, for further details see [19]. It is easy to see that $M_{a}$ possesses a superattracting fixed point at $z=0$ and a free critical point at $z=a$. When $a$ belongs to the basin of attraction of the origin, we say that this critical point has been captured. The connected components of the parameter space for which this phenomenon occurs are thus called capture zones. Define the main capture zone as the set of parameter values $a$ for which the free critical point belongs to the immediate basin of the origin. Roughly speaking, with the use of internal and external rays, Milnor showed how the dynamics near zero (conjugated to $\theta \mapsto 2 \theta$ ) meets the dynamics near infinity (conjugated to $\theta \mapsto 3 \theta)$ at the boundary of the immediate basin of attraction of the origin. In [21] Roesch extended Milnor's results to the family

$$
M_{a, m}(z)=z^{m+1}-\frac{m+1}{m} a z^{m} .
$$

We consider (1.1) as the transcendental version of Roesch's family in the following sense: for all nonzero values of $\lambda, F_{\lambda, m}$ has a superattracting fixed point at $z=0$, and a free simple critical point at $z=-m$.

The dynamical behavior of the free critical point will be crucial to describe the structure of the dynamical plane. Basically there are three possibilities. Either $z=-m$ is captured or it is associated to a Fatou component different from the basin of $z=0$, or it is not associated to any Fatou component (landing on a periodic repelling point, escaping to infinity, etc.).

In this paper we deal only with parameters in capture zones. The topological description of Julia sets for these parameters has been previously addressed in a more general setting in $[\mathbf{3}],[\mathbf{2 0}]$ where it has been 
shown that the Julia set consists of uncountable many path-components, also known as hairs, and possibly with pinchings (see Section 2 for exact definitions and results). Nevertheless, this topological description does not give a full understanding of the combinatorics in the Julia set, and this is precisely one of our main goals of the present work. In Proposition A we address the topological description of Julia sets. Next, we follow Milnor's approach and study how the dynamics near zero meets the dynamics of the tails of the Julia set near infinity. More specifically, in Theorem B we show how the pinchings occur at the boundary of the immediate basin of attraction of the origin. In order to present a clear exposition we restrict its formulation to a particular case and discuss its generalization in Theorem D. Finally, we completely describe accessible points in the Julia set from the Fatou set in Theorem C.

The outline of the paper is as follows: in Section 2 we list definitions and previous results concerning the topology of $J\left(F_{\lambda, m}\right)$ and the family $F_{\lambda, m}$. The statements of our main results are found at the end of the section. The proof of Proposition A is found in Section 3. Then, in Section 4 we prove Theorem B using a polynomial-like construction around the origin and symbolic dynamics. The polynomial-like construction rigourously explains how $F_{\lambda, 2}$ acts as $z \mapsto z^{2}$ around the origin. The proof of Theorem C is found in Section 5. Finally in Section 6 we provide a partial generalization of Theorem $\mathrm{B}$ by showing how $F_{\lambda, m}$ acts as $z^{m}$ around the origin for $m>2$.

Acknowledgements. The first and second author are both partially supported by the European network 035651-2-CODY. They are also supported by MEC and CIRIT through the grants MTM2008-01486 and 2005SGR-00550, respectively. The second author is also partially supported by MEC through the grant MTM2006-05849/Consolider (including a FEDER contribution). The third author is supported by CONACyT grant 59183, CB-2006-01.

We wish to thank K. Barański and L. Rempe for useful conversations that clarified to us their results cited here. We thank our home institutions and the Mathematics Department at Boston University, Departament de Matemàtiques at Universitat Autònoma de Barcelona and the Fields Institute for their hospitality while this work was in progress. Finally we would like to thank the referee for her/his comments and helpful suggestions that greatly improved the presentation and final formulation of several results. 


\section{Preliminaries and statement of the results}

\subsection{Julia sets of hyperbolic transcendental entire maps.}

Nowadays there exists a comprehensive study related to dynamics and topology of Julia sets for entire transcendental maps. Some of the early prime works dealt mainly with complex exponential family $E_{\lambda}(z)=\lambda e^{z}$, $[\mathbf{1 2}],[\mathbf{1 1}],[\mathbf{7}],[\mathbf{2 2}]$, while some generalizations to other entire transcendental maps were also found in $[\mathbf{1 2}]$ and more recently in $[\mathbf{3}],[\mathbf{2 0}]$. Among several other topological results, it has been shown that under certain assumptions, the Julia set of an entire transcendental map consists, succinctly, by a Cantor set of curves extending to infinity in a specific asymptotic direction, each one of them having a distinguished landing point, called the endpoint. All points in these curves, except for some particular endpoints, have forward orbits that tend to infinity along the curves.

A formal characterization of the structure of the Julia set for some entire transcendental maps appeared first in [12], where the authors introduced the notion of an $N$-Cantor bouquet. For our purposes, we review the dynamical construction here.

For a fixed $N \in \mathbb{N}$, denote by

$$
\Sigma_{N}=\left\{s=\left(s_{0} s_{1} s_{2} \ldots\right) \mid s_{j} \in\{0,1,2, \ldots, N-1\} \text { for each } j\right\},
$$

the space of one-sided infinite sequences of $N$ symbols and by $\sigma$ the right-shift map acting on $\Sigma_{N}$.

Definition 2.1. An invariant set $C_{N}$ of $J(f)$ is an $N$-Cantor bouquet if there is a homeomorphism $h: \Sigma_{N} \times[0, \infty) \rightarrow C_{N}$ that satisfies the following conditions:

(a) For the projection $\pi: \Sigma_{N} \times[0, \infty) \rightarrow \Sigma_{N}$,

$$
\pi \circ h^{-1} \circ f \circ h(s, t)=\sigma(s) .
$$

(b) For each $s \in \Sigma_{N}$,

$$
\lim _{t \rightarrow \infty} h(s, t)=+\infty
$$

(c) If $t>0$, then for each $s \in \Sigma_{N}$,

$$
\lim _{j \rightarrow \infty} f^{j}(h(s, t))=+\infty .
$$

Once $s \in \Sigma_{N}$ has been fixed, the curve $\{h(s, t) \mid t>0\}$ is called a tail and $h(s, 0)=z_{s}$ is its endpoint. The union of a tail with its endpoint is known as a hair associated to $s$ and in this case, we say that the hair lands at $z_{s}$. For examples of non-landing hairs that instead accumulate on an indecomposable continuum, see $[\mathbf{1 0}]$. 
In order to exemplify this definition, we sketch the construction of an $N$-Cantor bouquet for $E_{\lambda}(z)=\lambda e^{z}$, with $\lambda \in(0,1 / e)$. We refer the reader to the proof of Theorem 3.3 in [12] and Proposition 1.2 in [9] for more details. For these parameters there exists a unique attracting fixed point that traps the orbit of the unique asymptotic value $z=0$, therefore $E_{\lambda}$ is hyperbolic. The horizontal lines $I_{k}=\{z \in \mathbb{C} \mid \operatorname{Im}(z)=$ $(2 k+1) \pi\}, k \in \mathbb{Z}$, are infinitely many connected components of the preimage of the negative real line $\mathbb{R}^{-}$under $E_{\lambda}$. Denote by $M_{k}$ the open strip bounded by $I_{k}$ and $I_{k+1}$. A point $z \in J\left(E_{\lambda}\right)$ has a well defined itinerary $s=\left(s_{0}, s_{1}, \ldots\right)$ if and only if $E_{\lambda}^{k}(z) \in M_{s_{k}}, k \geq 0$. Fix $N$ and take constants $\xi$ and $\eta$ such that the image under $E_{\lambda}$ of each rectangle

$$
R_{k}=\{z \in \mathbb{C} \mid \xi<\operatorname{Re}(z)<\eta,(2 k+1) \pi<\operatorname{Im}(z)<(2 k+3) \pi\}
$$

contains $R=\cup R_{k}$ for $k=0,1, \ldots, N-1$. Since the exponential map is expansive on $R$ one can show that each point, with an orbit never escaping from $R$, has a unique itinerary in $\Sigma_{N}$ and belongs to $J\left(E_{\lambda}\right)$. The set of non-escaping points is shown to be homeomorphic to $\Sigma_{N}$ and constitute the endpoints of the bouquet.

Redefining constants $\xi$ and $\eta$, it is possible to obtain for each $s \in$ $\Sigma_{N}$ a sequence of rectangles $R_{s_{k}} \subset M_{s_{k}}$ with increasing real part and $R_{s_{k}} \subset E_{\lambda}\left(R_{s_{k-1}}\right)$. Similar arguments show the existence of a single point in $R_{s_{0}}$ with an orbit escaping to infinity from the right hand direction following $s$. A continuity argument shows that in fact there exists a continuous curve (or tail) inside $M_{s_{0}}$ with same dynamics. Appropriate pullbacks of the tail can be done to see how the tail limits to the left at the endpoint associated to $s$, giving thus a full hair.

As $\Sigma_{N+1}$ naturally contains $\Sigma_{N}$, it follows that $C_{N} \subset C_{N+1}$ for each $N$, and the set

$$
\mathcal{C}=\overline{\bigcup_{N \geq 0} C_{N}}
$$

is known as a Cantor bouquet. In Figure 1(a) the Cantor bouquet of an exponential map is shown, where all hairs land and extend to infinity to the right. The main result in $[\mathbf{1 2}]$ is that for critically finite entire maps that meet certain growth condition, the Julia set of $f$ contains a Cantor bouquet. This is the case for $E_{\lambda}$ with $0<\lambda<1 / e$ with a unique attracting fixed point and the Fatou set coinciding with its basin of attraction.

Surprisingly, it was shown in [1] that every Cantor bouquet associated to the exponential family for $0<\lambda<1 / e$, as for $\lambda \sinh z$ and $\lambda \cosh z$ (also with a completely invariant basins of attraction of a single fixed point), are in fact homeomorphic to a unique topological abstract model 
called straight brush. This remarkable result leads to an alternative definition of a Cantor bouquet as a set homeomorphic to a straight brush. Although this equivalence is used sometimes in the literature, it is not straightforward to argue that the Julia set of an entire transcendental map is homeomorphic to the straight brush.

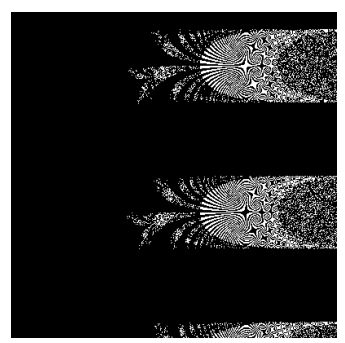

(a) An attracting fixed point for $\lambda=-0.05+1.14 i$.

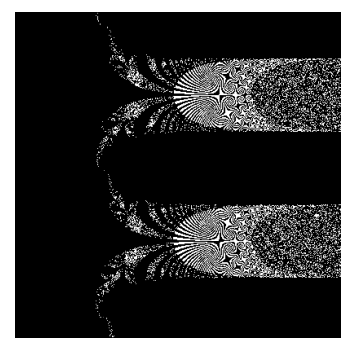

(b) An attracting 2-cycle for $\lambda=-4+1.14 i$.

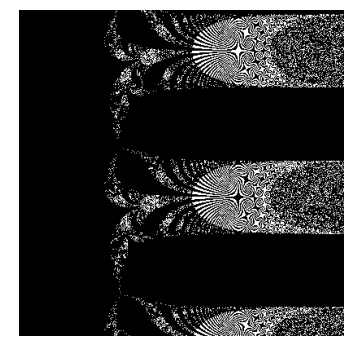

(c) An attracting 3-cycle for $\lambda=-1.06+1.89 i$.

Figure 1. The Julia set for $E_{\lambda}(z)=\lambda e^{z}$ is shown in white.

A key ingredient in the above descriptions of Julia sets is the existence of an attracting fixed point and the Fatou set consisting only of its immediate basin of attraction. With these assumptions, Barański studied in [4] the structure of Julia sets for more general maps. More precisely,

Theorem 2.2 (Barański, 2007). Let $f$ be an entire transcendental function of finite order so that all critical and asymptotic values are contained in a compact subset of a completely invariant attracting basin of a fixed point. Then $J(f)$ consists of disjoint hairs homeomorphic to the halfline $[0, \infty)$. In particular, all rays land on a distinguished endpoint.

Although Julia sets of entire transcendental functions may resemble a union of hairs, some endpoints may become the landing points of more than one hair. A multiple landing is commonly known as a pinching. For the exponential family, the existence of an attracting cycle of period two or higher provides sufficient conditions for pinchings, [5]. See Figures 1(b)-(c). In order to understand this new kind of Julia sets, a topological abstract model known as modified straight brush was given in $[6]$.

Recently, Rempe [20] has shown that these pinchings occur in dynamical planes for a large number of entire transcendental maps of finite order belonging to class $\mathcal{B}$ (that is, the class of entire transcendental 
maps where Sing $\left(f^{-1}\right)$ is a bounded set). This result is a consequence of a strong rigidity theorem on the escaping set of the map. The escaping set, $I(f)$, is the set of points whose orbits under $f$ tend to infinity. For an entire transcendental map, the Julia set coincides with the boundary of $I(f)$. It is worth noticing that all points on a tail belong to the escaping set, while some endpoints may have escaping orbits too.

Roughly speaking, the rigidity theorem states that, if $f, g \in \mathcal{B}$ are quasiconformally equivalent near infinity (that is, there exist quasiconformal maps $\phi, \psi: \mathbb{C} \rightarrow \mathbb{C}$ that satisfy $f \circ \phi=\psi \circ g$ in a neighborhood of infinity, [15]), then their escaping sets are quasiconformally conjugate near infinity. In light of this, Rempe was able to study Julia sets of hyperbolic maps in the class $\mathcal{B}$, that is, entire maps with all its singular values belonging to an attracting basin of a periodic point. For our purposes (in particular, the proof of Proposition A), we merge Theorem 1.4 and Theorem 5.2 in $[\mathbf{2 0}]$ into the following result.

Theorem 2.3 (Rempe, 2009). Let $f, g \in \mathcal{B}$ be two hyperbolic maps of finite order, quasiconformally equivalent near infinity. Assume also that Sing $\left(g^{-1}\right)$ is contained in a compact subset of the completely invariant basin of attraction of a fixed attracting point for $g$. Then $f$ and $g$ are conjugate on their sets of escaping points and the conjugacy extends to a continuous surjective map from $J(g)$ onto $J(f)$. In particular all hairs land, and pinchings may also happen.

\subsection{The family $\boldsymbol{F}_{\lambda, m}$.}

We state without proofs some results on the parameter and dynamical plane of $F_{\lambda, m}$ that we will strongly use in subsequent sections. Recall that $F_{\lambda, m}(z)=\lambda z^{m} e^{z}$. Clearly, there exists a superattracting fixed point at $z=0$ for all choices of $\lambda$ and $m \geq 2$. Denote its basin of attraction by

$$
A(0)=A_{\lambda, m}(0)=\left\{z \in \mathbb{C} \mid F_{\lambda, m}^{n}(z) \rightarrow 0 \text { as } n \rightarrow \infty\right\},
$$

and its immediate basin by $A^{*}(0)=A_{\lambda, m}^{*}(0)$. In [16] it is proved that $A^{*}(0)$ contains the disk $D_{\varepsilon}=\{z \in \mathbb{C}|| z \mid<\varepsilon\}$ where $\varepsilon$ depends on $\lambda$ and $m$. Moreover, $F_{\lambda, m}$ has also a free simple critical point at $z=-m$. With this in mind, define a capture zone as a connected component in parameter plane given by

$(2.2) \mathcal{C}_{m}^{k}=\left\{\lambda \in \mathbb{C} \mid F_{\lambda, m}^{k}(-m) \in A^{*}(0), F_{\lambda, m}^{j}(-m) \notin A^{*}(0), 0 \leq j \leq k-1\right\}$, for each $m \geq 2$ and $k \geq 0$. Any $\lambda \in \mathcal{C}_{m}^{k}$ is called a capture parameter. In particular

$$
\mathcal{C}_{m}^{0}=\left\{\lambda \in \mathbb{C} \mid-m \in A^{*}(0)\right\},
$$


is known as main capture zone. The next theorem gathers some of the most important results related to the family $F_{\lambda, m}$ and found also in [16].

Theorem 2.4. The following statements hold for all $m \geq 2$.

(a) The main capture zone is a bounded component. If $\lambda \in \mathcal{C}_{m}^{0}$, then $A(0)=A^{*}(0)$.

(b) $\mathcal{C}_{m}^{1}=\emptyset$. In other words, $F_{\lambda, m}(-m) \in A^{*}(0)$ if and only if $-m \in$ $A^{*}(0)$.

(c) $\mathcal{C}_{m}^{2}$ is an unbounded component extending into the left or right hand plane depending on $m$.

(d) For $k>2, \mathcal{C}_{m}^{k}$ has infinitely many connected components extending to infinity in an asymptotic direction.

(e) For $k \geq 2$, if $\lambda \in \mathcal{C}_{m}^{k}$ then $A(0)$ has infinitely many connected components. All these components, except $A^{*}(0)$, are unbounded.

In Figure 2 we illustrate the parameter plane of $F_{\lambda, m}$ for $m=2$ and 3. The main capture zone is drawn in blue while other capture zones are shown in red. The parameter values for which the orbit of the free critical point does not converge to zero (so, it is not captured) but it is bounded are drawn in orange. The parameter values for which the orbit of the free critical point is unbounded are drawn in black. If $m$ is even, capture zones extend to $+\infty$ as the real part of $\lambda$ tends to $+\infty$, whereas if $m$ is odd these strips extend to $-\infty$ as the real part of $\lambda$ tends to $-\infty$.

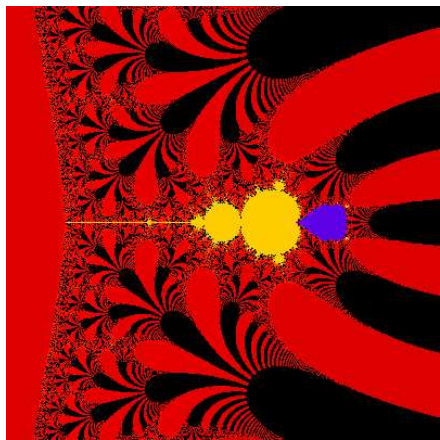

(a) Range $(-24,8) \times(-16,16)$.

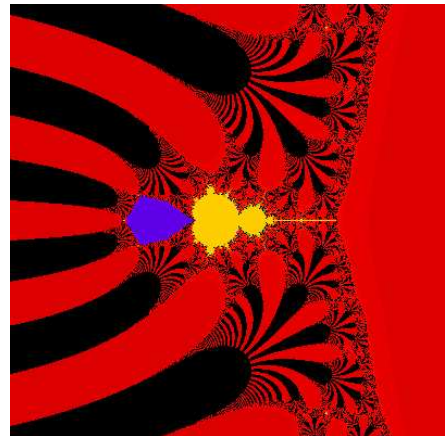

(b) Range $(-5,11) \times(-8,8)$.

Figure 2. Parameter plane for (a) $F_{\lambda, 2}$ and (b) $F_{\lambda, 3}$. Color codes are explained in the text. 
In Figures 3(a)-(c), we display the Julia set of $F_{\lambda, 2}$ for two different values of $\lambda$ (Figure 3(c) is a magnification of Figure $3(\mathrm{~b})$ ). The immediate basin of attraction of $z=0$ is shown in blue (although it is not visible in Figure $3(\mathrm{~b}))$. The connected components $A(0) \backslash A^{*}(0)$ are shown in red and the Julia set is in black. In Figure $3(\mathrm{a}) \lambda$ is drawn from $\mathcal{C}_{2}^{0}$ and the Julia set is a union of disjoint hairs. In Figures $3(\mathrm{~b})$ and (c) the $\lambda$ value belongs to $\mathcal{C}_{2}^{2}$ and the Julia set is a union of hairs with pinchings located at the boundary of the immediate basin of attraction of $z=0$ (and all its preimages).

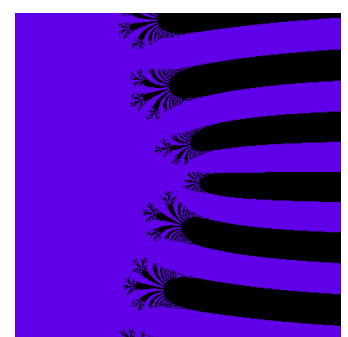

(a) $\lambda=0.32+0.82 i$.

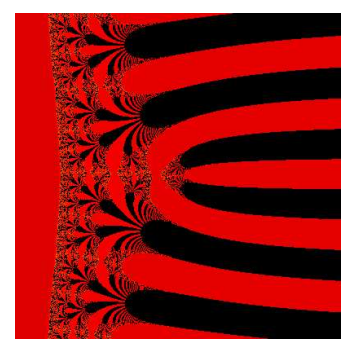

(b) $\lambda=-21+3 i$.

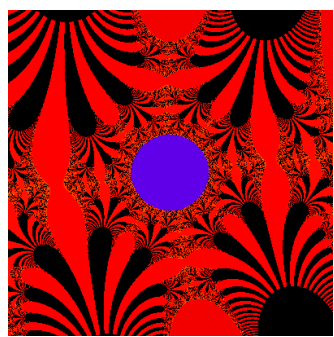

(c) $\lambda=-21+3 i$.

Range $(-15,15) \times(-15,15)$. Range $(-15,15) \times(-15,15)$. Range $(-.2, .2) \times(-.2, .2)$.

Figure 3 . The Julia set for $F_{\lambda, 2}$. Color codes are explained in the text.

2.3. Statement of the results. Throughout this work, we only deal with parameter values belonging to a capture zone. Under this assumption, it is straightforward to argue that the Fatou set coincides with the basin of attraction of $z=0$, and consequently, its complement is the Julia set. The first result follows almost immediately from Theorem 2.2 and Theorem 2.3.

Proposition A. Let $\lambda \in \mathcal{C}_{m}^{k}, m \geq 2, k \geq 0$. Then the Julia set of $F_{\lambda, m}$ is the union of hairs each one of them homeomorphic to $[0, \infty)$. Moreover, if $k=0$ the hairs are pairwise disjoint, while if $k \geq 2$ some of the hairs may share the same (non-escaping) endpoint.

Our second, and main result of this article, relates to the case $k \geq 2$, as we explain how the pinchings occur by matching the dynamics of the polynomial map $z \mapsto z^{m}$ with the dynamics of $z \mapsto \lambda e^{z}$. The bridge between these dynamical behaviors is build by means of symbolic dynamics and polynomial-like constructions, showing thus how tails of $F_{\lambda, m}$ land, 
in particular, at the boundary of the basin of attraction of $z=0$. See Figure 3(c).

For simplicity and clarity in the exposition, we state and prove the following result for particular values of $m$ and $k$. We have chosen them in order to capture the main arguments of the construction without introducing overwhelming notation. In Section 6 we state a more general case for arbitrary values of $m$ and $k$ and discuss the refinement of the previous arguments that will constitute its proof.

Theorem B. Let $F_{\lambda, 2}(z)=\lambda z^{2} e^{z}$ and assume that $\lambda \in \mathcal{C}_{2}^{3}$. Then, the boundary of $A^{*}(0)$ is a quasi-circle and $F_{\lambda, 2}$ restricted to $\partial A^{*}(0)$ is conjugate to $\theta \mapsto 2 \theta$ on the unit circle. Each point in the boundary of $A^{*}(0)$ is an endpoint of a hair. Moreover there exists a domain $\Gamma \subset \mathbb{C}$ such that

(a) If $F_{\lambda, 2}(-2) \notin \Gamma$, then each point in $\partial A^{*}(0)$ is a landing point of a unique hair, except the fixed point at $\partial A^{*}(0)$ and all its preimages, which are endpoints of exactly two hairs.

(b) If $F_{\lambda, 2}(-2) \in \Gamma$, then each point in $\partial A^{*}(0)$ is a landing point of a unique hair, except the two periodic points of period two on $\partial A^{*}(0)$ and all their preimages, which are endpoints of exactly two hairs.

Our third result addresses the question of accessible points in $J\left(F_{\lambda, m}\right)$ from the Fatou set. Let $f$ be an entire transcendental map. A point $z_{0}$ in $J(f)$ is accessible from the Fatou set if there is a continuous curve $\gamma:[0,1) \rightarrow \mathbb{C}$ for which $\gamma(t)$ lies in the Fatou set for all $t$ and $\lim _{t \rightarrow 1^{-}} \gamma(t)=$ $z_{0}$. Notice that such a curve must therefore lie in a single component of the Fatou set. The existence and characterization of non-accessible points is an interesting problem by itself that arises not only in the entire transcendental setting (see for example $[\mathbf{8}],[\mathbf{6}]$ ) but also in rational dynamics, [18]. For instance, in the exponential family $E_{\lambda}$, for $\lambda \in$ $(0,1 / e)$, the only accessible points are the endpoints. We can draw a result concerning the set of accessible points of the Julia set from the basin of attraction of 0 .

Theorem C. Let $m \geq 2$ and $k \geq 2$. The set of points in $J\left(F_{\lambda, m}\right)$ that are accessible can be completely characterized as follows.

(a) If $\lambda \in \mathcal{C}_{m}^{0}$, this set coincides with the set of all endpoints.

(b) If $\lambda \in \mathcal{C}_{m}^{k}$, this set coincides with the set of endpoints lying in $\partial A^{*}(0)$ and all its preimages. On the other hand, for any natural number $N$ there exists an $N$-Cantor bouquet that contains only nonaccessible points. 
Remark 2.5. Statement (a) in the above theorem has been already proved in $[\mathbf{3}]$. We include it here for completeness.

\section{Proof of Proposition A}

In Proposition $\mathrm{A}$, the case when $k=0$ follows directly from Theorem 2.2 , so we only consider $k \geq 2$. From Theorem 2.3 it follows that, if two maps are quasiconformally equivalent near infinity, then their escaping sets are homeomorphic. In our case, let $\lambda_{1} \in \mathcal{C}_{m}^{0}$ and $\lambda_{2} \in \mathcal{C}_{m}^{k}$ for some $k \geq 2$. Using $\phi_{1}(z)=\frac{\lambda_{2}}{\lambda_{1}} z$ and $\phi_{2}(z)=z$ we have that $\phi_{1} \circ F_{\lambda_{1}, m}=F_{\lambda_{2}, m} \circ \phi_{2}$, showing thus that the maps are quasiconformally equivalent. Thus, Theorem 2.3 implies the existence of a homeomorphism $\psi: I\left(F_{\lambda_{1}, m}\right) \rightarrow I\left(F_{\lambda_{2}, m}\right)$ between escaping sets that extends to a surjective continuous map

$$
\bar{\psi}: J\left(F_{\lambda_{1}, m}\right) \rightarrow J\left(F_{\lambda_{2}, m}\right) .
$$

Observe that the only points in $J\left(F_{\lambda_{1}, m}\right)$ which do not escape are contained in the set of endpoints (e.g., repelling periodic points). Thus, the homeomorphism $\psi$ may only extend to a surjective continuous map. Consequently $J\left(F_{\lambda_{2}, m}\right)$ consists only of landing hairs, and due to the lack of injectivity of $\bar{\psi}$, two or more endpoints in $J\left(F_{\lambda_{1}, m}\right)$ may be mapped to the same endpoint in $J\left(F_{\lambda_{2}, m}\right)$ and thus producing a pinching.

\section{Proof of Theorem B}

We begin by showing that the boundary of $A_{\lambda, 2}^{*}(0)$ is a quasi-circle using a polynomial-like construction (see [13] for an excellent exposition on polynomial-like mappings). After that, we use symbolic dynamics to show how the hairs land on $\partial A_{\lambda, 2}^{*}(0)$ and characterize the pinchings.

Before we start the proof, we describe a partition of the dynamical plane derived from the components of the preimage of $\mathbb{R}^{-}$for all $m \geq 2$ and any capture parameter $\lambda$. Hereafter, denote by $\operatorname{Arg}(\cdot) \in(-\pi, \pi]$ the principal argument. From the expression $F_{\lambda, m}(z)=\lambda z^{m} e^{z}$, it is easy to see that

$$
\operatorname{Arg}\left(F_{\lambda, m}(z)\right)=\operatorname{Arg}(\lambda)+m \operatorname{Arg}(z)+\operatorname{Im}(z) \quad(\bmod 2 \pi),
$$

so that, finding components of the preimage of $\mathbb{R}^{-}$is equivalent to solve $\operatorname{Arg}\left(F_{\lambda, m}(z)\right)=\pi$. Set $r=|z|$ and $\alpha=\operatorname{Arg}(z)$ so the above equation becomes

$$
r=\rho_{k}(\alpha)=\frac{(2 k+1) \pi-m \alpha-\operatorname{Arg}(\lambda)}{\sin (\alpha)}
$$


where $\alpha \in(-\pi, \pi)$ and $k \in \mathbb{Z}$. Thus, for fixed $\lambda$ and $m$, the components of the preimage of $\mathbb{R}^{-}$are given by

$$
\sigma_{k}=\rho_{k}(\alpha) e^{i \alpha} .
$$

In Figure 4 we show some of these curves for $m=5$. As their real parts tend to $+\infty$, all the $\sigma_{k}$ are asymptotic to the horizontal lines $\operatorname{Im}(z)=(2 k+1) \pi-\operatorname{Arg}(\lambda)$. There are $m$ of these curves that start at the origin (namely, $\sigma_{-j}, \ldots, \sigma_{j-1}$ when $m=2 j$, or $\sigma_{-j}, \ldots, \sigma_{j}$ when $m=2 j+1$ ), while all others start at $-\infty$.

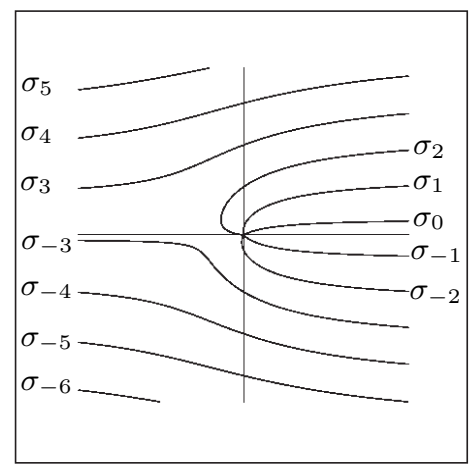

(a) Graphs of $\sigma_{k}$ for $m=5$.

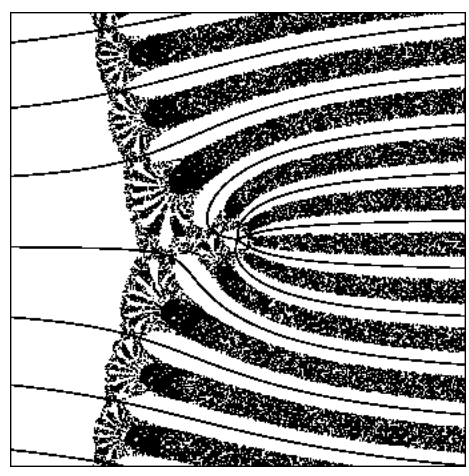

(b) The Julia set of $F_{\lambda, 5}$ lying over the graphs of $\sigma_{k}$.

FiguRE 4. Strips in dynamical plane.

The family of curves $\sigma_{k}, k \in \mathbb{Z}$, divides the plane into infinitely many regions or strips. One of these regions, denoted in what follows by $W$, contains $\mathbb{R}^{-}$and is bounded by four of these curves, namely, $\sigma_{j}, \sigma_{j-1}$, $\sigma_{-j}$ and $\sigma_{-(j+1)}$ when $m=2 j$, and by $\sigma_{j+1}, \sigma_{j}, \sigma_{-j}$ and $\sigma_{-(j+1)}$, when $m=2 j+1$. We say that $W$ has two arms in the far right-hand side plane and refer to the upper and lower arms of $W$ in the natural way. All other regions, denoted by $M_{k}$, are bounded by $\sigma_{k}$ and $\sigma_{k+1}$ with $k \neq j-1,-j-1$ if $m=2 j$, and $k \neq j,-j-1$ if $m=2 j+1$.

From the above construction, it is clear that

$$
F_{\lambda, m}: M_{k} \rightarrow \mathbb{C} \backslash \mathbb{R}^{-}
$$

is a bijective map for each $k$. Denote by

$$
L_{k}: \mathbb{C} \backslash \mathbb{R}^{-} \rightarrow M_{k}
$$


the inverse of $F_{\lambda, m}$ taking values in each $M_{k}$. In contrast, the map

$$
F_{\lambda, m}: W \rightarrow \mathbb{C} \backslash \mathbb{R}^{-}
$$

is a covering map of degree 2 , since contains the critical point $z=-m$ of multiplicity one. Before starting the polynomial-like construction, we need the following result.

Lemma 4.1. Let $\lambda$ be a parameter in $\mathcal{C}_{2}^{3}$. Then the Fatou component that contains $F_{\lambda, 2}(-2)$ does not intersect the negative real line.

Proof: We begin by assuming that $|\lambda|>e$. We first show that the immediate basin of attraction of $z=0$ is bounded, and consequently, if $U \subset F_{\lambda, 2}^{-1}\left(A^{*}(0)\right)$ denotes the connected component of the Fatou set that contains $F_{\lambda, 2}^{2}(-2)$, then $U$ is contained in a left-hand side plane.

An easy computation shows that if $|z|=1$ or if $\operatorname{Re}(z)=-1$, then $\left|F_{\lambda, 2}(z)\right|>1$. Since $z=0$ is a superattracting fixed point, there must be a preimage of the unit disk inside itself and containing the immediate basin. Moreover, since $U$ contains a neighborhood of $-\infty$, it cannot cross $\operatorname{Re}(z)=-1$ as points in this vertical line map outside $|z|=1$ while $F_{\lambda, 2}(U)$ is contained in $A^{*}(0)$.

Denote by $V$ the Fatou component in $F_{\lambda, 2}^{-1}(U)$ that contains $F_{\lambda, 2}(-2)$. We show that $V$ cannot cut across $\mathbb{R}^{-}$. Again, an easy computation shows that the image of $\mathbb{R}^{-} \cup\{0\}$ under $F_{\lambda, 2}$ is the segment from $z=0$ to $z=F_{\lambda, 2}(-2)$ contained in the straight line

$$
y_{1}(x)=\frac{\operatorname{Im}(\lambda)}{\operatorname{Re}(\lambda)} x, \quad x \in \mathbb{R} .
$$

Since $|\lambda|>e$, there exists a value $x_{0}=x_{0}(\lambda)<-1$ so the preimage of the unit circle that lies in $\mathbb{C} \backslash \overline{\mathbb{D}}$ can be parametrized by the curves

$$
y_{ \pm}(x)= \pm \sqrt{\frac{1}{|\lambda|} e^{-x}-x^{2}}, \quad x \leq x_{0},
$$

(see [16] for further details). Since $U$ maps inside the unit circle, it follows that $U$ lies to the left-hand side of the graphs $y_{ \pm}(x)$, and so $\operatorname{Re}(z)<x_{0}$ for all $z \in U$. If $\operatorname{Re}\left(F_{\lambda, 2}(-2)\right)>x_{0}$, then $V$ cannot cut across $\mathbb{R}^{-}$since for every $x \leq 0$, either $0 \leq \operatorname{Re}\left(F_{\lambda, 2}(x)\right)$ (if $\operatorname{Re}(\lambda) \geq 0$ ), or $x_{0}<\operatorname{Re}\left(F_{\lambda, 2}(-2)\right) \leq \operatorname{Re}\left(F_{\lambda, 2}(x)\right)<0$ (if $\operatorname{Re}(\lambda)<0$ ), and thus $F_{\lambda, 2}(x) \notin U$. 
Assuming $\operatorname{Re}\left(F_{\lambda, 2}(-2)\right) \leq x_{0}$, then

$$
\left|\operatorname{Im}\left(F_{\lambda, 2}(-2)\right)\right|>y_{+}\left(\operatorname{Re}\left(F_{\lambda, 2}(-2)\right)\right) .
$$

We claim that the straight line $y_{1}(x)$ cuts the graph of $y_{ \pm}(x)$ only once and from the above inequality, this crossing occurs at some $x<$ $\operatorname{Re}\left(F_{\lambda, 2}(-2)\right)$. Indeed, it can be verified that the equation

$$
\frac{1}{|\lambda|} e^{-x}-x^{2}=\left(\frac{\operatorname{Im}(\lambda)}{\operatorname{Re}(\lambda)}\right)^{2} x^{2},
$$

has a unique solution for $x<-1$ when $|\lambda|>e$, and consequently $V$ has an empty intersection with $\mathbb{R}^{-}$.

Observe that the boundedness of $A^{*}(0)$ does not depend on the condition $|\lambda|>e$. By Theorem 2.4, all connected components $\mathcal{C}_{2}^{3}$ are unbounded, thus a standard argument of continuous dependence of Julia sets on the parameter $\lambda$ moving inside a hyperbolic component shows that $A^{*}(0)$ must remain bounded for all parameter values in $\mathcal{C}_{2}^{3}$. Similar arguments as those given above show that $U$ is contained in the left-hand side plane and the result follows suit for all $\lambda \in \mathcal{C}_{2}^{3}$.

Proposition 4.2. Let $\lambda$ be a parameter in $\mathcal{C}_{2}^{3}$ and $r>0$ large enough. Then there exist open, bounded and simply connected domains $U_{r}$ and $V_{r}$ of $\mathbb{C}$ such that $0 \in \overline{U_{r}} \subset V_{r}$ and satisfying $\left(F_{\lambda, 2}, U_{r}, V_{r}\right)$ is a polynomiallike mapping of degree 2. Moreover, the filled Julia set of $\left(F_{\lambda, 2}, U_{r}, V_{r}\right)$ is a quasi-disk and coincides with $\overline{A_{\lambda, 2}^{*}(0)}$.

Proof: Since $\lambda \in \mathcal{C}_{2}^{3}$, we have that $F_{\lambda, 2}^{3}(-2)$ lies in the immediate basin of 0 . Consider a non-closed simple curve completely contained in $A_{\lambda, 2}^{*}(0)$, joining 0 and $F_{\lambda, 2}^{3}(-2)$ and coinciding with an straight line inside $D_{\varepsilon}$ (ref. Section 2.2). Pulling back this curve twice, it defines a simple curve $\gamma$ in the Fatou set joining $+\infty$ and $F_{\lambda, 2}(-2)$ that cuts across each straight line $\operatorname{Re}(z)=M$ once, for sufficiently large $M>0$. The proof is divided in three steps, and the third step in two cases.

The first step is to consider the preimage of $\gamma$ in $W$. By Lemma 4.1 it follows that $\gamma \cap \mathbb{R}^{-}=\emptyset$, so each connected component of the preimage of $\gamma$ belongs to one of the $M_{i}$ or to $W$. Now, since $z=-2$ is a simple critical point, the preimage of $\gamma$ consists of two simple curves that meet only at that point. Denote these curves by $\alpha$ and $\beta$ and note that they extend to $+\infty$ along a different arm of $W$. To fix notation, let $\alpha$ be the curve extending along the lower arm and $\beta$ the one extending in the upper arm. 


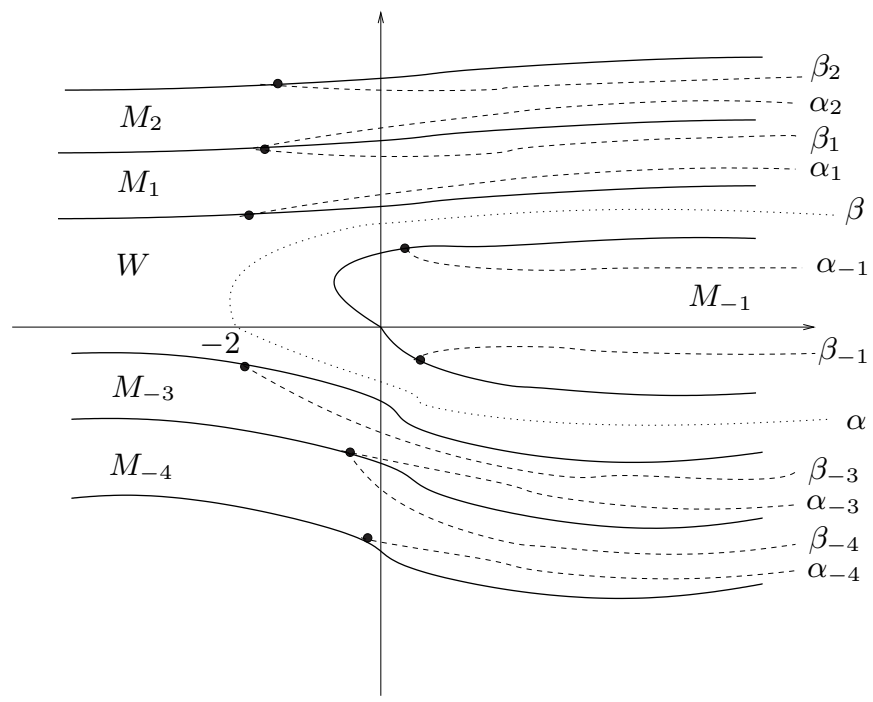

Figure 5. Dynamical plane of $F_{\lambda, 2}$ divided into fundamental domains. The curves $\alpha$ and $\beta$ are drawn in dotted lines. The components of the preimages of $\alpha$ and $\beta$ in each $M_{k}$, denoted by $\alpha_{k}$ and $\beta_{k}$, are drawn in dashed lines.

The second step is to take the components of the preimage of $\alpha \cup \beta$ in $W$ and on each fundamental domain $M_{k}$. Clearly, $F_{\lambda 2}^{-1}(-2)$ consists of infinitely many points, denoted by $q_{k}$, and each one lying in a unique $\sigma_{k}$ curve. On the other hand, using the inverse branches $L_{k}$ in (4.2), we get exactly one preimage of $\alpha$ and one preimage of $\beta$ in each domain $M_{k}$. We denote them by $\alpha_{k}$ and $\beta_{k}$, respectively. By continuity, $\alpha_{k}$ and $\beta_{k}$ are joined at the corresponding point $q_{k}$. See Figure 5 . The components of the preimages of $\alpha$ and $\beta$ in $W$ are obtained as follows. Since $F_{\lambda, 2}$ is a covering map of degree 2 in $W$, components of the preimages consist of four curves denoted by $\alpha_{W}^{i}$ and $\beta_{W}^{i}, i=1,2$, extending to $+\infty$ through the lower $(i=1)$ and upper $(i=2)$ arms of $W$.

The third step in the polynomial-like mapping construction is to define the sets $U_{r}$ and $V_{r}$ of the statement with the desired properties. We denote by $\Gamma$ the connected component of the complement of $\alpha \cup \beta$ containing the origin. The proof splits in two possible scenarios depending on the location of $F_{\lambda, 2}(-2)$ with respect of $\Gamma$. 
Case 1. $F_{\lambda, 2}(-2) \notin \Gamma$. Far to the right, the relative position of $\alpha$ and $\beta$ with respect to $\left\{\alpha_{W}^{1}, \beta_{W}^{1}, \alpha_{W}^{2}, \beta_{W}^{2}\right\}$ when going from bottom to top of $\Gamma$ is either $\left\{\alpha, \alpha_{W}^{1}, \beta_{W}^{1}, \beta, \alpha_{W}^{2}, \beta_{W}^{2}\right\}$ if $F_{\lambda, 2}(-2)$ lies below $\alpha$, or $\left\{\alpha_{W}^{1}, \beta_{W}^{1}, \alpha, \alpha_{W}^{2}, \beta_{W}^{2}, \beta\right\}$ if $F_{\lambda, 2}(-2)$ lies above $\beta$. To see the claim suppose that $F_{\lambda, 2}(-2)$ lies below $\alpha$ (the second case is similar). Let $L$ be a vertical segment far to the right and contained in the lower arm of $W$. By construction, its bottom and top endpoints are in $\sigma_{-2}$ and $\sigma_{-1}$ respectively, so $F_{\lambda, 2}(L)$ is a simple curve that surrounds the origin starting and ending at $\mathbb{R}^{-}$. As we move in $L$ from bottom to top, $F_{\lambda, 2}(L)$ travels in a counterclockwise direction, cutting $\gamma, \alpha$ and $\beta$ in this order. Their components of the preimage located in the lower arm of $W$ are ordered as $\alpha, \alpha_{W}^{1}$ and $\beta_{W}^{1}$. For the upper arm of $W$ the arguments are similar. See Figure 6(a).

For any $r>0$ sufficiently large, define $V_{r}=\{z \in \Gamma \mid \operatorname{Re} z<r\}$ and let $U_{r}$ be the connected component of the preimage of $V_{r}$ containing the origin. Then $U_{r}$ is bounded by pieces of $\left\{\alpha_{W}^{1}, \beta_{W}^{1}, \alpha_{-1}, \beta_{-1}\right\}$ plus two (almost) vertical lines. Clearly $\bar{U}_{r} \subset V_{r}$ and $F_{\lambda, 2}$ maps $\partial U_{r}$ onto $\partial V_{r}$ in a 2-to-1 fashion. Thus $\left(F_{\lambda, 2}, U_{r}, V_{r}\right)$ is the desired polynomial-like map of degree 2. In Figure 7(a) the sets $U_{r}$ and $V_{r}$ are illustrated.

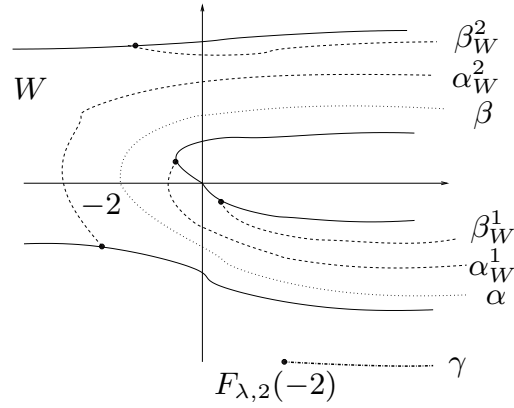

(a) Case 1 in the proof of Proposition 4.2 .

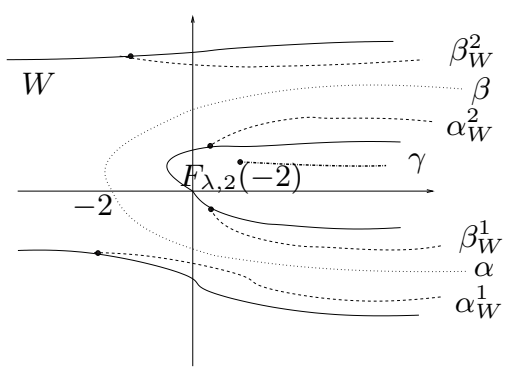

(b) Case 2 in the proof of Proposition 4.2 .

FiguRE 6 . Sketch of the relative positions of $\alpha$ and $\beta$ with respect to their preimage components in $W$.

Case 2. $F_{\lambda, 2}(-2) \in \Gamma$. Since $F_{\lambda, 2}(-2)$ belongs to the Fatou set and takes two iterates to be in $A^{*}(0)$, we have that $F_{\lambda, 2}(-2)$ must belong to one of the two connected components of $F_{\lambda, 2}^{-2}\left(A^{*}(0)\right)$ in $\Gamma$. In general for each $k \geq 2$ and $m \geq 2, F_{\lambda, m}^{-k}\left(A^{*}(0)\right)$ consists of finitely many unbounded and connected components extending towards $\infty$ when $m$ is even, or $-\infty$ when $m$ is odd. We name these type of components fingers. 


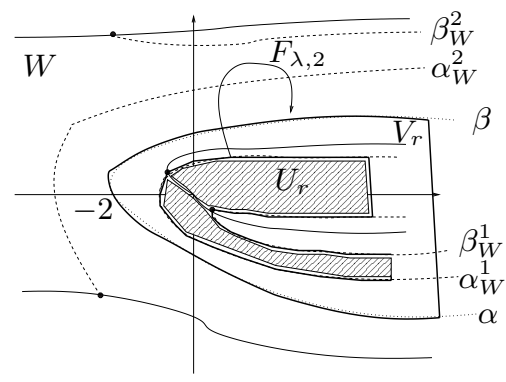

(a) Polynomial-like construction for $m=2$ and $k=3$ when $F_{\lambda, 2}(-2) \notin \Gamma$.

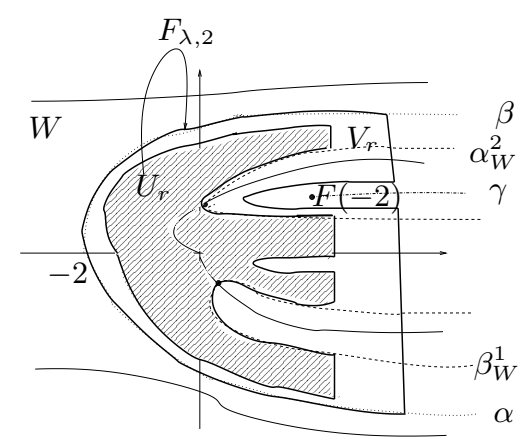

(b) Polynomial-like construction for $m=2$ and $k=3$ when $F_{\lambda, 2}(-2) \in \Gamma$.

Figure 7. Domains $U_{r}$ and $V_{r}$ in Proposition 4.2.

Denote by $\mathcal{F}$ the finger where $\gamma$ is located. Then, far to the right, the relative position of the curves $\alpha$ and $\beta$ with respect to $\left\{\alpha_{W}^{1}, \beta_{W}^{1}, \alpha_{W}^{2}, \alpha_{W}^{2}\right\}$ when going from bottom to top of $\Gamma$ is $\left\{\alpha_{W}^{1}, \alpha, \beta_{W}^{1}, \alpha_{W}^{2}, \beta, \beta_{W}^{2}\right\}$ (regardless of the finger where $\gamma$ is located). See Figure 6(b). The above claim follows easily from similar arguments as in Case 1.

Let $r$ be a positive real number such $r>1+\operatorname{Re}\left(F_{\lambda, 2}(-2)\right)$ and $\gamma \cap\{z \mid$ $\operatorname{Re}(z)=r\}$, which is a single point by the construction of $\gamma$. We define $\tilde{B}_{\varepsilon}(\gamma)$ to be a closed $\varepsilon$-neighborhood of a piece of $\gamma$, completely contained in $\mathcal{F}$. More precisely,

$$
\tilde{B}_{\varepsilon}(\gamma)=\{z \in \mathcal{F} \mid \operatorname{Re}(z) \leq r \text { and } \operatorname{dist}(z, \gamma) \leq \varepsilon\},
$$

where $\operatorname{dist}(z, \gamma)$ denotes the natural distance between compact sets. Let $B_{\varepsilon}(\gamma)$ be the union of $\tilde{B}_{\varepsilon}(\gamma)$ and, if they exist, the bounded components of the complement of $\tilde{B}_{\varepsilon}(\gamma)$, so that $B_{\varepsilon}(\gamma)$ is simply connected.

Let now $V_{r}=\{z \in \Gamma \mid \operatorname{Re}(z)<r\} \backslash B_{\varepsilon}(\gamma)$ and define $U_{r}$ as the connected component of the preimage of $V_{r}$ containing the origin. We claim that, for $r>0$ large enough, $\left(F_{\lambda, 2}, U_{r}, V_{r}\right)$ is the desired polynomial-like mapping of degree 2. To see this, we study the preimage of the boundaries of $V_{r}$ in $\Gamma$. The preimage of the arcs of $\alpha$ and $\beta$ that bound $V_{r}$ are arcs of the curves $\beta_{W}^{1}, \alpha_{-1}, \beta_{-1}$ and $\alpha_{W}^{2}$. The preimage of $B_{\varepsilon}(\gamma)$ in $\Gamma$ has two connected components: one is a connected component contained in $M_{-1}$, and the other is a connected component contained in the intersection of $\Gamma$ with a small neighborhood of a piece of $\alpha \cup \beta$. Finally, the preimage of $\Gamma \cap\{z \mid \operatorname{Re} z=r\}$ is the suitable union of (almost) vertical lines that bound $U_{r}$ from the right. This construction is 
illustrated in Figure 7(b). Since $\overline{U_{r}} \subset V_{r}$ and the map $F_{\lambda, 2}: U_{r} \rightarrow V_{r}$ sends $\partial U_{r}$ to $\partial V_{r}$ with degree 2, we conclude that for $r$ large enough, the triple $\left(F_{\lambda, 2}, U_{r}, V_{r}\right)$ is a polynomial-like mapping of degree 2.

Our final step is to show that the filled Julia set of $\left(F_{\lambda, 2}, U_{r}, V_{r}\right)$ is a quasi-disk. By the Straightening Theorem, $[\mathbf{1 3}]$, there exists a quasiconformal mapping $\varphi$ that conjugates $F_{\lambda, 2}$ to a polynomial $Q$ of degree 2 on the set $U_{r}$. That is $\left(\varphi \circ F_{\lambda, 2} \circ \varphi^{-1}\right)(z)=Q(z)$ for all $z \in U_{r}$. Since $z=0$ is a superattracting fixed point for $F_{\lambda, 2}$ and $\varphi$ is a conjugacy, we have that $z=0$ is superattracting for $Q$. Hence, after perhaps a holomorphic change of variables, we may assume that $Q(z)=z^{2}$. Thus, the filled Julia set of $\left(F_{\lambda, 2}, U_{r}, V_{r}\right)$ given by

$$
K\left(F_{\lambda, 2}\right)=\left\{z \in U_{r} \mid F_{\lambda, 2}^{n}(z) \in U_{r} \text { for all } n\right\}=\bigcap_{n \geq 0} F_{\lambda, 2}^{-n}\left(U_{r}\right),
$$

coincides with $\overline{A^{*}(0)}$ and is the image under the quasi-conformal map $\varphi^{-1}$ of the closed unit disk. So $\partial A^{*}(0)$ is a quasi-circle. Using $\varphi$ we can parametrize $\partial A^{*}(0)$ so that any point $z \in \partial A^{*}(0)$ can be written as $z=\varphi^{-1}(\theta)$, or simply $z=z_{\theta}$, for some $\theta \in \mathbb{S}^{1}$. Since $\varphi$ conjugates $F_{\lambda, 2}$ on $\partial A^{*}(0)$ with the map $\theta \mapsto 2 \theta$ on $\mathbb{S}^{1}$, we have that $F_{\lambda, 2}\left(z_{\theta}\right)=z_{2 \theta}$.

An important consequence of the previous proposition is that the only points in $U_{r}$ that never escape this domain under forward iteration are precisely the points in $\overline{A_{\lambda, 2}^{*}(0)}$, which is a quasi-disk. By construction, all points in $\partial A_{\lambda, 2}^{*}(0)$ do belong to the Julia set but do not belong to the escaping set, so they must be endpoints of at least one hair. Thus we have

Corollary 4.3. Each point in $\partial A_{\lambda, 2}^{*}(0)$ is the landing point of at least one hair in the Julia set.

What remains to prove involves the use of symbolic dynamics that will allow us to show how pinchings occur at the boundary of $A_{\lambda, 2}^{*}(0)$. As stated in Theorem $\mathrm{B}$, these pinchings will depend on the relative position of $F_{\lambda, 2}(-2)$ with respect to the boundaries of $\Gamma$.

Case 1. $F_{\lambda, 2}(-2) \notin \Gamma$. Fix $r$ large enough and let $\Omega$ be the connected component of the preimage of $\Gamma$ containing 0 . Observe that $\Omega$ is the union of $U_{r}$ and two strips $H_{i}, i=0,1$ extending to infinity in the asymptotic direction. Also $\partial \Omega$ (as well as $\partial \Gamma$ ) belongs to the Fatou set. By the above corollary, there are uncountable many landing points in the interior of $U_{r}$, and thus, uncountably many hairs crossing the righthand side boundary of $U_{r}$ (or equivalently, the left-hand side boundary of $H_{i}$ ). 
Next step is to characterize hairs with endpoints in the boundary of $A^{*}(0)$. If one of those hairs has an endpoint in $U_{r}$ but not in $\partial A^{*}(0)$, it must eventually escape from $\Omega$ since by Proposition 4.2, the only points in the Julia set with forward orbits completely contained inside $U_{r}$ are the points in $\partial A^{*}(0)$. Consequently hairs with endpoints in $\partial A^{*}(0)$ must remain under forward iteration inside $\Omega$ and, respectively, their tails must also remain in $H_{0} \cup H_{1}$. Since each $H_{i}$ maps one-to-one into $\Gamma \backslash V_{r}$ we can associated to each of those tails an itinerary $s \in \Sigma_{2}$ in the natural way. And viceversa, for each sequence $s \in \Sigma_{2}$ there is a unique tail in $\Omega$ with this itinerary. Indeed, the existence and uniqueness of a tail with prescribed itinerary can be guaranteed by the action of $F_{\lambda, 2}$ over the left-hand side boundary of $H_{i}$, which cuts across each $H_{i}$ in an almost vertical line for all $r$ sufficiently large. And observing that far to the right $F_{\lambda, 2}$ behaves like the exponential map, continuity arguments (similar to those described in Section 2 for an $N$-Cantor bouquet) guarantee the existence of tails associated to itineraries in $\Sigma_{2}$.

To finish the proof, note that each of the endpoints in $\partial A^{*}(0)$ has dynamics governed by $\theta \mapsto 2 \theta$ and the tails have associated a unique sequence $s \in \Sigma_{2}$. This will determine how many tails land on each endpoint. Indeed, there are two fixed tails associated to $\overline{0}$ and $\overline{1}$, and a unique fixed point in $\partial A^{*}(0)$, so both fixed tails must land on it giving two pinched hairs. The preimage of these hairs in $\Omega$ contains two new hairs (with sequences $1 \overline{0}$ and $0 \overline{1}$ ) landing at the preimage point of the fixed point in $\partial A^{*}(0)$, thus again given a pinching. Clearly, this type of pinching occurs at each point in the backward orbit of the fixed point restricted to $\partial A^{*}(0)$.

Any other point not contained in the backward orbit of the fixed point has a unique tail landing on it. To see this, let $z \in \partial A^{*}(0)$ be one of those points and set $\theta=\varphi(z)$. Then, the itinerary for $\theta$ in $\mathbb{S}^{1}$ is given by its binary expansion whereas from the construction above, the tail landing on $z$ must share the same itinerary with respect to $H_{0}$ and $H_{1}$. Since there is a unique tail associated to an itinerary $s \in \Sigma_{2}$, the result follows.

Case 2. $F_{\lambda, 2}(-2) \in \Gamma$. Select $r>0$ large enough and let $\Omega$ be the connected component of the preimage of $\Gamma$ containing 0 , minus an $\varepsilon$-neighbourhood of a piece of $\gamma$, as in (4.3). Observe that $\Omega$ is the union of $U_{r}$ and four arms $H_{i}, i=0, \ldots, 3$, extending to infinity in the asymptotic direction. We know that on the right-hand side boundary of $U_{r}$ there are infinitely hairs crossing it with landing points inside $U_{r}$, since the boundary of $\Omega$ belongs to the Fatou set. As before, the hairs with 
endpoints in $U_{r}$ but not in $\partial A^{*}(0)$ must eventually escape under forward iteration from $\Omega$, and hairs with endpoints in $\partial A^{*}(0)$ must remain in $\Omega$, so their tails must remain in $\cup H_{i}$. However, in this case, the dynamics on those hairs are governed by a subshift of four symbols, since each strip fails to cover $\cup H_{i}$ under the action of $F_{\lambda, 2}$.

Labeling the strips in an increasing order from bottom to top, the transition matrix of the subshift is given by

$$
M=\left(\begin{array}{llll}
0 & 0 & 0 & 1 \\
1 & 1 & 1 & 0 \\
0 & 0 & 0 & 1 \\
1 & 1 & 1 & 0
\end{array}\right)
$$

where $a_{i j}=1$ (respectively, $a_{i j}=0$ ) means the strip $H_{i}$ covers (respectively, does not cover) $H_{j}$. For instance, there is a unique fixed sequence $\overline{1}$ and four period two sequences given by $\overline{03}, \overline{30}, \overline{23}$ and $\overline{32}$. Denote by $\Sigma_{M}$ the space of allowed sequences generated by $M$.

As before, we can associate to any tail in $\cup H_{i}$ with endpoint in $\partial A^{*}(0)$ a sequence in $\Sigma_{M}$ and viceversa. To finish the proof, note that each of the endpoints in $\partial A^{*}(0)$ has dynamics governed by $\theta \mapsto 2 \theta$. We construct a partition in $\partial A^{*}(0)$ to determine how many tails land on each endpoint.

From the transition matrix is easy to see the existence of a unique fixed tail (with itinerary $\overline{1}$ ) that in turn, must land at the unique fixed point in $\partial A^{*}(0)$. Now, we can compute periodic points of period two in $\partial A^{*}(0)$. Under the angle doubling map restricted to $\mathbb{S}^{1}$, these points are $\theta=1 / 3$ and $\theta=2 / 3$. Using the conjugacy $\varphi^{-1}$, denote the corresponding 2 periodic points in $\partial A^{*}(0)$ by $z_{1 / 3}$ and $z_{2 / 3}$. As mentioned before, the four tails of period two have sequences $\overline{03}, \overline{30}, \overline{23}$ and $\overline{32}$. An easy combinatorial argument shows that the tails associated to $\overline{03}$ and $\overline{32}$ land at a single endpoint of period two whereas the tails associated to $\overline{30}$ and $\overline{23}$ land in the other periodic point. The set of periodic points of period two and their preimages, namely $z_{1 / 6}, z_{1 / 3}, z_{2 / 3}$ and $z_{5 / 6}$ defines the desired partition on $\partial A^{*}(0)$. To match the sequences in $\Sigma_{M}$ with this partition, we label them in the following way: traveling along $\partial A^{*}(0)$ in a counterclockwise direction, associate the symbol 0 to the arc joining $z_{2 / 3}$ and $z_{5 / 6}$, the symbol 1 to the arc joining $z_{5 / 6}$ and $z_{1 / 6}, 2$ to the arc joining $z_{1 / 6}$ and $z_{1 / 3}$, and 3 to the arc joining $z_{1 / 3}$ and $z_{2 / 3}$. We left to the reader to verify that under $F_{\lambda, 2}$ the arc with symbol 0 covers the arc with symbol 3 and so on. Hence, the transition matrix for this partition is exactly $M$.

As before, each point disjoint from the backward orbits of $z_{1 / 3}$ and $z_{2 / 3}$ has a unique itinerary $s \in \Sigma_{M}$, and by uniqueness of the tails, this point 
is the landing point of a single tail associated to $s$. This concludes the proof of Theorem B.

\section{Proof of Theorem C}

In this section we prove our result concerning accessibility of points in the Julia set. Part (a) follows directly from Theorem $\mathrm{C}$ in [3], since when $\lambda$ belongs to $\mathcal{C}_{m}^{0}, F_{\lambda, m}$ has a completely invariant basin of attraction.

Part (b) is shown as follows. First, we claim that a point $z_{0}$ in the Julia set is accessible if and only if it belongs to the boundary of some Fatou component. Assume first that $z_{0}$ belongs to the boundary of some connected component $U$ in the Fatou set. From the polynomiallike construction (see Proposition 4.2) $\bar{U}$ is a quasi-disk, so there exists a quasi-conformal map $\varphi: \bar{U} \rightarrow \overline{\mathbb{D}}$. Setting $\theta_{0}=\operatorname{Arg}\left(\varphi\left(z_{0}\right)\right)$, the curve $\gamma(t)=\varphi^{-1}\left(t \cdot e^{i \theta_{0}}\right), t \in[0,1)$, is an accessible path for $z_{0}$. The reverse implication is straightforward.

There are points in $J\left(F_{\lambda, m}\right)$ that are not accessible, as they do not belong to the boundary of any connected component of the Fatou set. In particular any point whose orbit escapes to infinity is non-accessible, and those points are dense in the Julia set. Moreover, any repelling periodic point that does not belong to $\partial A^{*}(0)$ must be non-accessible. In fact, we show that for any integer $N \geq 0$, there exists a non-accessible and forward invariant $N$-Cantor bouquet contained in $J\left(F_{\lambda, m}\right)$.

We use the same notation as in Proposition 4.2. Consider the components of the preimage of $\alpha \cup \beta$ in all fundamental domains outside $\Gamma \cup W$. These components bound simply connected $C$-shaped regions, denoted by $D_{k}$, and induce a natural alphabet $\mathcal{A}=\{ \pm 1, \pm 2, \ldots, \pm k, \ldots\}$. Following the $N$-Cantor bouquet construction reviewed in Section 2, we see that, for each natural number $N$, the set $C_{N}$ given by

$$
C_{N}=\left\{z \in J\left(F_{\lambda, m}\right) \mid F_{\lambda, m}^{n}(z) \in \bigcup_{|k| \leq N} D_{k} \text { for all } n \in \mathbb{Z} \text { and } k \in \mathcal{A}\right\},
$$

is an $N$-Cantor bouquet of non-accessible points. Indeed, the fundamental domains $D_{k}$, with $k \in \mathcal{A},|k| \leq N$ define in a natural way an itinerary $s=\left(s_{0}, s_{1}, \ldots\right), s_{i} \in \mathcal{A}$ and $\left|s_{i}\right| \leq N$ for all points in $C_{N}$. For a fixed $s$, the only point with bounded orbit and itinerary $s$ is the endpoint, while those points with unbounded orbit and itinerary $s$ form the tail of the hair.

Since all accessible points must lie in the boundary of a Fatou component (and thus eventually enter the domain $\Gamma \cup W$ under iteration), is now straightforward to see that every point in $C_{N}$ is non-accessible. 


\section{Generalizations}

In the proof of Theorem B we used two main tools: polynomial-like construction and symbolic dynamics. In order to extend our results for any $m \geq 2$ and $k \geq 2$, we start with the following proposition that generalizes the polynomial-like construction.

Proposition 6.1. For $m \geq 2, k \geq 2$, let $r>0$ large enough and assume that $\lambda \in \mathcal{C}_{m}^{k}$ and $\gamma \cap \mathbb{R}^{-}=\emptyset$. Then, there exist open, bounded and simply connected domains $U_{r}$ and $V_{r}$ of $\mathbb{C}$, such that $0 \in \overline{U_{r}} \subset V$ and satisfying that $\left(F_{\lambda, m}, U_{r}, V_{r}\right)$ is a polynomial-like mapping of degree $m$. Moreover, the filled Julia set of $\left(F_{\lambda, m}, U_{r}, V_{r}\right)$ is a quasi-disk and coincides with $\overline{A_{\lambda, m}^{*}(0)}$.

The proof of the above result splits into two cases depending once more on the relative position of the free critical value with respect to $\Gamma$. In the case when $F_{\lambda, m}(-m)$ does not lie in $\Gamma$, the same arguments as in Proposition 4.2 follow through. In the case when $F_{\lambda, m}(-m)$ lies in $\Gamma$, the arguments of the polynomial-like construction are again similar, nevertheless we must take into account the orbit of the critical value with respect to $\Gamma$. Let $k^{\prime}$ denote the number of iterates that takes $F_{\lambda, m}(-m)$ to leave $\Gamma$ for the first time. Since $\lambda$ is a capture parameter, $1 \leq k^{\prime} \leq k-2$. Then, after removing $k^{\prime} \varepsilon$-neighborhoods around $\gamma$, $F_{\lambda, m}(\gamma), \ldots, F_{\lambda, m}^{k^{\prime}-1}(\gamma)$ in the definition of $V_{r}$, the argument now follows as in Proposition 4.2.

In the light of Proposition 6.1 there exist $m-1$ fixed points in $\partial A_{\lambda, m}^{*}(0)$ since the dynamics in $\partial A_{\lambda, m}^{*}(0)$ is now conjugated to $\theta \mapsto m \theta$. When $F_{\lambda, m}(-m) \notin \Gamma$ the same arguments as in the proof of Theorem B, case (a), imply the existence and uniqueness of $m$ fixed tails. From these ideas, we conclude

Theorem D. Let $m \geq 2, k \geq 2$ and assume that $\lambda \in \mathcal{C}_{m}^{k}$ and $F_{\lambda, m}(-m) \notin$ $\Gamma$. Then, the boundary of $A^{*}(0)$ is a quasi-circle and each one of its points is an endpoint. Also, $F_{\lambda, m}$ on $\partial A^{*}(0)$ is conjugate to $\theta \mapsto m \theta$ on the unit circle. Moreover, each point in $\partial A^{*}(0)$ is a landing point of a unique hair, except for a single fixed point in $\partial A^{*}(0)$ and all its preimages, which are endpoints of exactly two hairs.

\section{References}

[1] J. M. Aarts and L. G. Oversteegen, The geometry of Julia sets, Trans. Amer. Math. Soc. 338(2) (1993), 897-918. 
[2] I. N. Baker, J. Kotus, And L. Yinian, Iterates of meromorphic functions. IV. Critically finite functions, Results Math. 22(3-4) (1992), 651-656.

[3] K. BARAŃski, Trees and hairs for some hyperbolic entire maps of finite order, Math. Z. 257(1) (2007), 33-59.

[4] W. Bergweiler, Invariant domains and singularities, Math. Proc. Cambridge Philos. Soc. 117(3) (1995), 525-532.

[5] R. Bhattacharjee and R. L. Devaney, Tying hairs for structurally stable exponentials, Ergodic Theory Dynam. Systems 20(6) (2000), 1603-1617.

[6] R. Bhattacharjee, R. L. Devaney, R. E. L. Deville, K. JoSić, And M. Moreno Rocha, Accessible points in the Julia sets of stable exponentials, Discrete Contin. Dyn. Syst. Ser. B 1(3) (2001), 299-318.

[7] C. Bodelón, R. L. Devaney, M. Hayes, G. Roberts, L. R. Goldberg, and J. H. Hubbard, Hairs for the complex exponential family, Internat. J. Bifur. Chaos Appl. Sci. Engrg. 9(8) (1999), 1517-1534.

[8] R. L. Devaney and L. R. Goldberg, Uniformization of attracting basins for exponential maps, Duke Math. J. 55(2) (1987), 253-266.

[9] R. L. Devaney and X. Jarque, Misiurewicz points for complex exponentials, Internat. J. Bifur. Chaos Appl. Sci. Engrg. 7(7) (1997), 1599-1615.

[10] R. L. Devaney, X. Jarque, and M. Moreno Rocha, Indecomposable continua and Misiurewicz points in exponential dynamics, Internat. J. Bifur. Chaos Appl. Sci. Engrg. 15(10) (2005), 3281-3293.

[11] R. L. Devaney and M. Krych, Dynamics of $\exp (z)$, Ergodic Theory Dynam. Systems 4(1) (1984), 35-52.

[12] R. L. Devaney and F. Tangerman, Dynamics of entire functions near the essential singularity, Ergodic Theory Dynam. Systems 6(4) (1986), 489-503.

[13] A. Douady and J. H. Hubbard, On the dynamics of polynomiallike mappings, Ann. Sci. École Norm. Sup. (4) 18(2) (1985), 287-343.

[14] A. È. ERËMenKo AND M. Yu. Lyubich, Iterations of entire functions, (Russian), Dokl. Akad. Nauk SSSR 279(1) (1984), 25-27; English translation in: Soviet Math. Dokl. 30(3) (1984), 592-594. 
[15] A. È. Erëmenko and M. Yu. Lyubich, Dynamical properties of some classes of entire functions, Ann. Inst. Fourier (Grenoble) 42(4) (1992), 989-1020.

[16] N. Fagella And A. Garijo, Capture zones of the family of functions $\lambda z^{m} \exp (z)$, Internat. J. Bifur. Chaos Appl. Sci. Engrg. 13(9) (2003), 2623-2640.

[17] L. R. Goldberg and L. Keen, A finiteness theorem for a dynamical class of entire functions, Ergodic Theory Dynam. Systems 6(2) (1986), 183-192.

[18] C. MCMullen, Automorphisms of rational maps, in: "Holomorphic functions and moduli", Vol. I (Berkeley, CA, 1986), Math. Sci. Res. Inst. Publ. 10, Springer, New York, 1988, pp. 31-60.

[19] J. W. Milnor, On cubic polynomial maps with periodic critical point, Part I, Preprint, Stony Brook Institute for Mathematical Sciences (2008), available at: http://www.math.sunysb.edu/ jack/.

[20] L. REMPE, Rigidity of escaping dynamics for transcendental entire maps, Acta Math. (to appear), arXiv:math 0605058v3 (2009).

[21] P. Roesch, Puzzles de Yoccoz pour les applications à allure rationnelle, Enseign. Math. (2) 45(1-2) (1999), 133-168.

[22] D. Schleicher AND J. Zimmer, Escaping points of exponential maps, J. London Math. Soc. (2) 67(2) (2003), 380-400.

\section{Antonio Garijo:}

Departament d'Enginyeria Informàtica i Matemàtiques

Universitat Rovira i Virgili

Tarragona, Catalunya

Spain

E-mail address: antonio.garijo@urv.cat

Xavier Jarque:

Departament de Matemàtica Aplicada i Anàlisi

Universitat de Barcelona

Barcelona, Catalunya

Spain

E-mail address: xavier.jarque@ub.edu

Mónica Moreno Rocha:

Centro de Investigación en Matemáticas

Guanajuato

Mexico

E-mail address: mmoreno@cimat.mx

Primera versió rebuda el 8 de setembre de 2008, darrera versió rebuda el 16 de juny de 2009. 